\title{
Biomaterials and Regenerative Medicine in Ophthalmology: Second Edition
}

\author{
Edited by Traian V. Chirila and Damien G. Harkin \\ Publisher: Woodhead Publishing, Elsevier
}

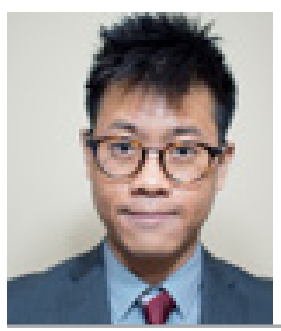

William Ngo, OD, PhD, FAAO

UAB School of Optometry

The University of Alabama at Birmingham

Birmingham, Alabama, USA

\footnotetext{
$\mathrm{B}$
} iomaterials have wide and diverse applications in the various disciplines within medicine. These "natural material emulators" are used to construct artificial joints, stents, implants, biosensors, contact lenses, and drug-delivery systems. The development and engineering of biomaterials are extremely complex, and require an understanding of both the laboratory and clinical sciences to fully appreciate. This book is part of the Woodhead Publishing Series in Biomaterials, a collection of books (115 books) that discuss biomaterials in nearly every medical application.

This book reviews the scientific literature, and the development and applications of biomaterials in ophthalmology. Based on a consideration of the specific roles biomaterials have within the various parts of the eye, this book is structured into four major parts:

1) Materials, properties, and considerations. This section provides a brief overview of hydrogels and their design, considerations, and applications in contact lenses and vitreous substitutes. Biomaterials used in intraocular drugdelivery and their associated challenges are also discussed.

2) Biomaterials for the repair and regeneration of the cornea and ocular surface, and 3) for the repair and regeneration of the retina. These two sections discuss the use of biomaterials to restore tissues and functions of the ocular surface and retina. The former section elaborates on the different types of scaffolds that have been designed for the regeneration and reconstruction of corneal tissue. Limbal stem cell deficiency is discussed, along with the use of contact lenses to deliver stem cells to restore the ocular surface. The latter section discusses the nature and challenges of transplanting scaffolds, retinal ganglion cells, retinal pigment epithelium, and pluripotent cells.

4) Other applications of biomaterials in ophthalmology. This section mainly discusses design and biocompatibility concepts regarding implants and prostheses, intraocular lenses, specialized glaucoma drug-delivery devices, and artificial corneal tissues.

This book has achieved its goal of reviewing the applications of biomaterials and regenerative medicine in ophthalmology. It reviews and reports on the main findings and results of various scientific studies, which range from in vitro laboratory experiments to experimental case studies. Each chapter begins with a brief introduction to provide relevant background information, and concludes with a discussion of future trends and challenges associated with the topic. As can be expected in a review, the specific details of the methods and procedures used in these studies are not provided. However, this does not pose much of a problem since all sources are cited. This book will be wellsuited for those who are looking to gain a modern perspective on the application of biomaterials and regenerative medicine in ophthalmology. $\bullet$ 